\title{
Novedad empírica y creación de conceptos
}

\section{Roberto Torretti}

\begin{abstract}
Resumen
Debido a la historicidad de la razón, más que inventariar sus principales conceptos en un momento dado nos interesa estudiar el proceso de su formación y fijación. En este artículo se ilustra ese proceso con ejemplos tomados de la historia de la física. El primer ejemplo concierne a la subordinación en el siglo XVII de los fenómenos archiconocidos de la caída libre y el movimiento de los planetas a un concepto nuevo; los restantes, tomados de la electrodinámica del siglo XIX y la microfísica y la cosmología del siglo XX, ilustran el juego mutuo de tales conceptos con novedades empíricas que en algunos casos los provocan, en otros los realizan.

Palabras clave: razón, formación de conceptos científicos, gravedad, electromagnetismo, relatividad especial, átomo, cosmología, relatividad general, expansión del universo, radiación cósmica de fondo, Galileo, Newton, Faraday, Maxwell, Hertz, Lorentz, Poincaré, Einstein, Rutherford, Hubble, Friedmann, Lemaître, Penzias, Wilson.
\end{abstract}

\section{Empirical novelty and concept creation}

\begin{abstract}
Due to the historical origin and development of reason, an inventory of its main concepts at a particular moment is less interesting to us than the study of their formation and fixation. This process is studied here in the light of examples from the history of physics. The first one concerns the subsumption of the well-known phenomena of free fall and planetary motion to a new concept of gravity in the 17th century; the remaining examples, drawn from 19th century electrodynamics
\end{abstract}


and 20th century microphysics and cosmology, display the interplay of such concepts with empirical novelties that prompt them in some case and realize them in others.

Keywords: reason, scientific concept formation, gravity, electromagnetism, special relativity, atom, cosmology, general relativity, expansion of the universe, cosmic background radiation, Galileo, Newton, Faraday, Maxwell, Hertz, Lorentz, Poincaré, Einstein, Rutherford, Hubble, Friedmann, Lemaître, Penzias, Wilson.

Kant nos persuadió de que no hay percepción sin conceptos que unifiquen la variedad de las impresiones sensibles y las ordenen en el sistema de la experiencia. Pero, a diferencia de Kant, no nos cabe pensar que nuestra naturaleza humana - incluidas las facultades de concebir, juzgar y razonar- haya caído del cielo, armada de pies a cabeza. Fruto de la evolución de las especies por mutación aleatoria y selección natural, el Homo sapiens vive desde su primera aparición sobre la Tierra una historia en el curso de la cual se suceden y desplazan unas a otras formas diversas y aun discrepantes de comunicación, de pensamiento, de vida. Bajo esta perspectiva, un inventario (como el intentado por Kant en su tabla de categorías y su lista de Ideas de la razón) de los principales conceptos corrientes, pero seguramente variables, interesa menos que una reflexión filosófica sobre los procesos, no necesariamente uniformes, de creación y fijación de tales conceptos.

El surgimiento de los conceptos que operaron en los albores del pensamiento humano se pierde en la llamada noche de los tiempos y solo con gran dificultad e incertidumbre es posible discernir y explorar sus huellas en las lenguas vivas o en aquellas que no hablamos pero que todavía sobreviven en la condición de lenguas muertas. En particular, la gramática y el léxico de las lenguas indogermánicas es una fuente de instrucción incesante sobre la mentalidad neolítica que todavía nos embebe y puede ser una ayuda para emanciparnos de ella. ${ }^{1}$ Con todo, los intentos para extraer del griego o del sánscrito conocimientos sustantivos sobre los conceptos primigenios de la humanidad en general o de nuestra particular cultura acaban siendo bastante especulativos, como bien sabemos los lectores de Heidegger. Así por ejem-

\footnotetext{
${ }^{1}$ Onians (1951) y Benveniste (1969) utilizan activa y eficazmente su conocimiento de las antiguas lenguas indoeuropeas para arrojar luz sobre las fuentes de la racionalidad euroamericana.
} 
plo, que el verbo $\dot{\alpha} \lambda \eta \theta \varepsilon v ́ \omega^{2}$ signifique "decir la verdad" sugiere que en alguna etapa de su historia, alguna comunidad influyente de griegohablantes (o de pregriegohablantes) entendió que - al menos en cierto importante grupo de casos - cuando se dice la verdad, lo dicho deja de estar oculto o encubierto; pero aunque esto efectivamente hubiera sido así, ello no implicaría que 'desencubrimiento' o 'desocultación' sea el significado invariable, o genuino, o siquiera preferible de 'verdad'.

Evidentemente, la historia de las ciencias ofrece al interesado en los procesos de creación de conceptos un campo de estudios mucho mejor documentado que el inicio de las lenguas. En particular, la física moderna ha sido, durante los últimos cuatro siglos el escenario de numerosas innovaciones intelectuales, más o menos drásticas, que han facilitado una mejor comprensión de muchísimos fenómenos, algunos familiares, como los movimientos de la Luna, pero otros inesperados y sorprendentes, como la radioactividad o la fuga de las galaxias, o, como el alternador eléctrico o las ondas hertzianas, fabricados en el laboratorio para conferir realidad a un concepto nuevo. En las páginas siguientes examinaré una serie de ejemplos tomados de la historia de la física. El primero envuelve la subordinación de fenómenos archiconocidos a un concepto nuevo; los restantes ilustran el juego mutuo de tales conceptos con novedades empíricas que en algunos casos los provocan, en otros los realizan.

En el siglo XVII, Galileo propuso un modelo matemático simple para el fenómeno de la caída de los cuerpos sobre la Tierra: si acordamos ignorar la resistencia del aire, la caída vertical de cualquier cuerpo realiza un movimiento uniformemente acelerado; la aceleración constante es independiente del peso y la índole del cuerpo. El modelo fue aplicado con éxito a la descripción y predicción del movimiento de los proyectiles, que Galileo concibe como resultante del movimiento uniforme y rectilíneo con que el proyectil sale, por

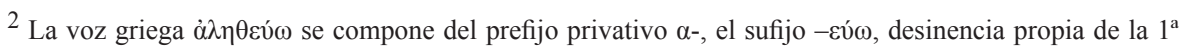
persona singular del presente indicativo de muchos verbos, y la raíz $\lambda \eta \theta-$, común a muchas formas del verbo $\lambda \alpha v \theta \alpha ́ v \omega$, 'ocultarse, escapar a percepción o detección' (de hecho, $\lambda \eta \dot{\theta} \theta \omega$ es presente del indicativo en Ilíada, 13.273, 23.323; Edipo Rey, 1325).

${ }^{3}$ Hay, con todo, un texto clásico que al parecer documenta precisamente la elucidación de $\alpha \dot{\lambda} \eta \dot{\theta} \theta \varepsilon 1 \alpha$ por Heidegger. En el conocido pasaje dónde Tucídides (1.23.6) presenta el motivo que habría hecho prácticamente inevitable la guerra entre los peloponesios y los atenienses que es el tema de su obra, se utili-

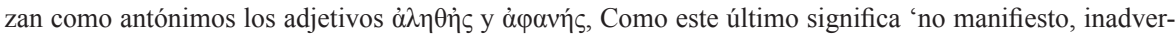
tido, secreto, invisible', el primero significa 'manifiesto, evidente, patente, obvio'. 'H $\mu \dot{\varepsilon} v \dot{\alpha} \lambda \eta \theta \varepsilon \varepsilon \sigma \tau \dot{\alpha} \tau \eta$

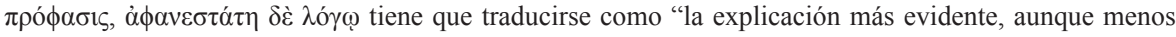
manifestada en palabras".
} 
ejemplo, de la boca de un cañón, en la dirección en que este apunta, y de la aceleración vertical constante que le imprime la gravedad desde el instante de su salida. ${ }^{4}$ Newton hizo extensivo este concepto al movimiento de la Luna, ${ }^{5}$ con una modificación decisiva: la aceleración vertical —es decir, dirigida hacia el centro de la Tierra - no puede ser la misma a cualquier altura, sino que varía inversamente como el cuadrado de la distancia entre ese centro y el cuerpo acelerado. (La modificación es razonable si suponemos con Newton que toda aceleración es el efecto de una fuerza; si esta es ejercida desde el centro de la Tierra sobre los cuerpos que la rodean, es verosímil que se diluya según crece la esfera donde se ejerce, la cual es proporcional al cuadrado de su radio). La Luna no se estrella, como el proyectil, contra la Tierra, sino que la circunda indefinidamente, porque en cada momento su velocidad en la dirección perpendicular al radio que la une al centro de la Tierra es suficiente para mantenerla en órbita. En octubre de 1957, 270 años después de la publicación de los Principios de Newton, Sputnik I, el primer satélite artificial, fue puesto en órbita por la U.R.S.S., brindando una realización práctica (Bachelard diría "fenomenotécnica"-vide Torretti 2011) del concepto newtoniano: un proyectil lanzado con tal velocidad que, en vez de caer a la Tierra, describe una trayectoria elíptica en torno a ella.

Newton concibió la aceleración de gravedad como efecto de una fuerza de atracción de la materia sobre la materia. Postuló que la fuerza entre dos cuerpos es directamente proporcional a la cantidad de materia o "masa" de cada uno. ${ }^{6}$ Habiendo demostrado que modelos construidos conforme a este concepto representan satisfactoriamente los movimientos de los satélites de Júpiter en torno a este planeta y de los planetas en torno al Sol, Newton concluyó que entre todos los cuerpos actúa una fuerza directamente proporcional a sus masas e inversamente proporcional al cuadrado de sus distancias. Esta audacísima generalización valió a Newton el dístico de Pope:

Nature and Nature's Laws lay hid in Night:

God said, "Let Newton be!" and all was light.?

\footnotetext{
${ }^{4}$ Galileo Galilei (1638), pp. 150ss; en Galileo (1964), vol. 8, pp. 190ss.

${ }^{5}$ Los primeros cálculos de Newton basados en esta idea datan de 1666. La hizo pública en su obra maestra editada en 1687.

${ }^{6}$ Como el efecto acelerador de una fuerza es — conforme al segundo Axioma del Movimiento de Newton- inversamente proporcional a la masa del cuerpo acelerado, la contribución de esta a la fuerza se elimina de la ecuación; por tanto, dicha masa no afecta la velocidad ni la trayectoria de la caída.

7 "La naturaleza y las leyes de la naturaleza yacían ocultas en la noche./ Dios dijo: ‘¡Hágase Newton!’ y todo fue luz” (Epitafio para la tumba de Isaac Newton en la Abadía de Westminster; en Pope 1963, p. 808).
} 
A fines del siglo XVIII el concepto newtoniano de atracción a distancia conforme a una relación cuantitativa simple había sido extendido analógicamente desde la gravedad a otras dos clases de fuerza, aparentemente menos ubicuas que aquella, pero conocidas desde antaño: la fuerza magnética con que se atraen o repelen los extremos de dos agujas imantadas, y la fuerza

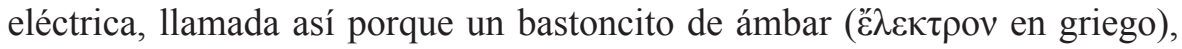
frotado vigorosamente con un paño, atrae cuerpecillos ligeros, por ejemplo, trocitos de papel. Los chinos descubrieron durante el primer milenio de nuestra era que uno de los extremos de una aguja imantada apunta al norte y el otro al sur. Basaron en este hallazgo la invención de la brújula como instrumento de navegación. Cualquiera que haya jugado con dos brújulas ha visto que los extremos de idéntica orientación se repelen y los de orientación opuesta se atraen. A mediados del siglo XVIII, Benjamín Franklin introdujo una clasificación homóloga de las fuerzas eléctricas, llamando electricidad vidriosa (vitreous) o positiva a la ejercida desde un trozo de vidrio que se ha frotado contra un trozo de resina, y electricidad resinosa o negativa a la ejercida desde el trozo de resina que se frotó contra aquel. Hoy entendemos que el frotamiento traslada del vidrio a la resina partículas cargadas de electricidad negativa, alterando así el equilibrio entre los dos tipos de electricidad que reinaba en ambos cuerpos antes de frotarlos. Coulomb (1784) postuló que los cuerpos cargados con el mismo tipo electricidad se repelen y que los cargados con electricidad de tipo opuesto se atraen con una fuerza proporcional a la magnitud de sus respectivas cargas e inversamente proporcional al cuadrado de las distancias. $\mathrm{Al}$ año siguiente, Coulomb propuso para las fuerzas magnéticas una ley de atracción y repulsión de la misma forma. Pocos años antes, Galvani había generado por primera vez una corriente eléctrica, al conectar casualmente las patas de una rana a dos metales diferentes. Galvani entendió que los efectos observados manifestaban la fuerza vital de la rana. Esta interpretación fue desestimada en cuanto Volta fabricó la primera pila eléctrica, capaz de generar una corriente eléctrica que fluye por un alambre conductor entre los dos extremos de una pila de discos alternados de cobre y de zinc, empapados con agua salada. Un par de décadas más tarde, el físico danés Christian Oersted, convencido - por la filosofía de Schelling - del parentesco íntimo entre todas las fuerzas naturales, se puso a investigar una posible interacción entre los fuerzas eléctricas y las magnéticas. Mientras intentaba en vano que una aguja imantada perpendicular a un alambre por el cual fluía una corriente se alinease con este, descubrió sin buscarlo el efecto contrario: una aguja paralela a un alambre conductor gira 90 grados al circular corrien- 
te por este. Este descubrimiento dio paso a la espectacular conversión de la electricidad de juguete de salón en pilar de la civilización planetaria. En este proceso fue decisiva la obra de Michael Faraday, de la que tomo mi segundo ejemplo de innovación conceptual, seguida esta vez inmediatamente por su realización en el laboratorio.

Para describirlo emplearé la noción de campo de fuerzas que Faraday introducirá muchos años después. Si coloco uno o más imanes sobre una mesa y riego limaduras de hierro en el espacio que los separa, estas se ordenarán en líneas tangentes en cada punto a la dirección de la fuerza magnética ejercida sobre la limadura presente en ese punto. Faraday entenderá que estas líneas son la expresión visible de la dirección e intensidad de las fuerzas magnéticas que en cada momento llenan el espacio. Paralelamente a este campo de fuerzas magnéticas, supuso que existe un campo de fuerzas eléctricas, organizado también en "líneas de fuerza". Oersted mostró que la variación del campo eléctrico debida al movimiento de cargas eléctricas en un alambre conductor origina un campo magnético que, prevaleciendo sobre el campo magnético de la Tierra, hace girar a las brújulas en la vecindad del alambre. En 1821 Faraday inventó un dispositivo donde una corriente eléctrica, mediante el efecto descubierto por Oersted, imprime a un cuerpo un movimiento de rotación; esto es, un rudimentario motor eléctrico, un artefacto que, como diríamos hoy, convierte energía eléctrica en energía mecánica. Faraday se atrevió a pensar que también debía producirse el efecto inverso: la conversión de energía mecánica en energía eléctrica. Una variación del campo magnético producida moviendo imanes debería originar un campo eléctrico que pondría en movimiento las cargas eléctricas situadas en ese campo variable, generando una corriente en un alambre conductor. Guiado por esta idea, Faraday fabricó en 1831 el primer prototipo de generador eléctrico o dinamo, el llamado disco de Faraday. Este era, como el rotor de 1821, demasiado ineficiente para tener utilidad práctica. ${ }^{8}$ Pero los generadores utilizados hoy están concebidos conforme a esa misma idea.

En los ejemplos que he considerado hasta ahora, un concepto disponible fue aplicado con éxito a casos empíricos nuevos por la vía de modificar una de sus condiciones o extenderlo analógicamente. Mis dos próximos ejemplos

\footnotetext{
${ }^{8}$ Se suele escuchar que, cuando alguien preguntó a Faraday de que servían sus juguetes (el rotor, o el generador, o ambos), él habría respondido “¿De qué sirve un recién nacido?” Pero I.B. Cohen (1987) ha mostrado que Faraday usó la frase, dando debido crédito por ella a Benjamin Franklin, en una conferencia de 1816 "Sobre el oxígeno, el cloro, el yodo y el flúor”. Cf. asimismo Chapin (1985).
} 
envuelven una situación diferente: el concepto que se intenta aplicar a la experiencia es una consecuencia lógica del sistema conceptual aceptado. En el primer caso que examinaremos, esto da lugar a una laboriosa búsqueda que culmina en la realización experimental del concepto. En el segundo, la realización predicha no se logra y el resultado negativo de los experimentos pertinentes provoca una innovación intelectual que, en el caso que propondré, fue revolucionaria. En ambos casos, el concepto en cuestión es una consecuencia de la teoría de los fenómenos electromagnéticos propuesta por James Clerk Maxwell (1861/62, 1864).

Maxwell organizó sistemáticamente los hallazgos de Faraday en una teoría matemática de gran originalidad y elegancia. Aunque es imposible hacerle justicia mediante una sumaria presentación verbal, las indicaciones que ofreceré a continuación permitirán entender, espero, mis dos ejemplos. Siguiendo a Faraday, Maxwell concibe un doble campo de fuerzas, eléctricas y magnéticas, que cubre todo el espacio. La intensidad y dirección de las dos fuerzas presentes en cada punto varían con el tiempo de acuerdo con las ecuaciones de Maxwell, que expresan la interdependencia entre los dos campos y su dependencia de la densidad de la carga eléctrica y el flujo de carga eléctrica. Las ecuaciones de Maxwell implican que cualquier perturbación local de los campos se propaga en todas direcciones mediante ondas electromagnéticas (análogamente a como se propaga la perturbación causada en la superficie de un estanque de agua por una piedra que cae en él). Las ecuaciones asignan un determinado valor constante, representado tradicionalmente por la letra $c$, a la velocidad de propagación de estas ondas en el vacío (en otros medios, como el aire, el agua, el vidrio, esa velocidad es algo menor). Al comprobar que $c$ concordaba con la velocidad de la luz dentro de un margen de error de 1,5\% (a la sazón admisible), Maxwell concluyó que la luz consistía en ondulaciones transversales del mismo medio que es la causa de los fenómenos eléctricos y magnéticos?.

${ }^{9}$ La siguiente imagen ofrece un modelo visual de una onda electromagnética simple en un instante dado. (Las ondas reales son superposiciones de muchas ondas simples).

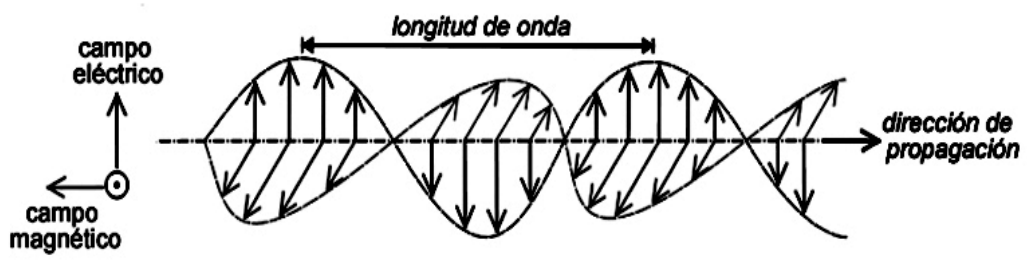

Las flechas representan la intensidad y dirección del campo correspondiente en el punto de partida de las mismas. Con el trascurso del tiempo, la onda avanza en la dirección de propagación con una velocidad 
En su trabajo de 1861/62, Maxwell intentó sin éxito concebir las fuerzas eléctricas y magnéticas como tensiones en un cuerpo de índole peculiar, carente de masa, que llenaría todo el espacio, permeando los cuerpos materiales ordinarios: el éter. En su trabajo de 1864, Maxwell renuncia a este propósito y se contenta con establecer que un fenómeno gobernado por sus ecuaciones satisface en todo caso el Principio de Hamilton, reconocido como principio fundamental de la mecánica. Al mostrar que sus ecuaciones de campo satisfacen las exigencias de este principio, Maxwell prueba que los fenómenos electromagnéticos descritos o predichos por las ecuaciones pueden explicarse por el comportamiento mecánico de una sustancia peculiar que permea todos los cuerpos ordinarios y llena completamente el espacio que los separa; pero no nos ofrece ni siquiera un barrunto de cómo podría desarrollarse tal explicación mecánica. Durante el resto del siglo XIX y hasta la publicación en 1905 del artículo de Einstein sobre la electrodinámica de los cuerpos en movimiento, los físicos comulgaron todos con la realidad del éter, imperceptible y elusivo.

La teoría de Maxwell fue pronto aceptada en los países de habla inglesa, más lentamente en Europa continental. Como no había motivo alguno para suponer que las ondas del éter estuviesen confinadas a las frecuencias de las ondas luminosas (que obviamente están determinadas por la receptividad de nuestro sentido de la vista), se imponía la tarea de buscar ondas electromagnéticas de otras frecuencias. Heinrich Hertz, el primer partidario entusiasta de la teoría de Maxwell en Alemania, decidió procurarle un decisivo respaldo experimental produciendo tales ondas en su laboratorio. En 1887, Hertz anunció sus primeros resultados. ${ }^{10}$ Utilizando lo que ahora llamamos un circuito LC o circuito resonante, había producido una oscilación eléctrica que inducía una oscilación homóloga en otro circuito desconectado del primero y situado en otro lugar de su laboratorio. Había fabricado un fenómeno nuevo, jamás observado hasta entonces en la naturaleza, pero que realizaba un concepto implicado por la teoría de Maxwell. Este hallazgo daría lugar a la revolución en la comunicaciones ocurrida en el siglo XX. En 1895, Gugliel-

\footnotetext{
que en el vacío es igual a $c$, esto es, 299.792.458 metros por segundo (este número es exacto, pues en él se basa, desde 1983, la definición del metro). Conviene recordar que la velocidad de propagación de una onda es igual al producto de su longitud (la distancia entre dos crestas consecutivas en la misma dirección) por su frecuencia (el número de oscilaciones por unidad de tiempo).

${ }^{10}$ Hertz (1887), reproducido en Hertz (1892), pp. 32ss. y, en traducción inglesa, en Hertz (1893), pp. 29ss. Buchwald (1995) ofrece un estudio detallado de la historia de este descubrimiento.
} 
mo Marconi logró trasmitir una señal eléctrica a 2400 metros de distancia. En 1900 patentó la telegrafía sin hilos. En diciembre de 1901 trasmitió una señal desde Cornwall, en Inglaterra, a Terranova, en Canadá.

Mi segundo ejemplo es, como dije, un resultado negativo que para la ciencia física fue mucho más sorpresivo y fecundo que el anterior. Si la luz se propaga en el éter en cierta dirección con velocidad $c$, su velocidad con respecto a un cuerpo que se mueve en el éter con velocidad $v$ en la dirección contraria tendría que ser $c+v$. Como la Tierra se mueve alrededor del Sol en direcciones opuestas en los dos solsticios, o en los dos equinoccios, no puede reposar en el éter durante todo el año. Como su velocidad de traslación es de aproximadamente $30 \mathrm{~km} / \mathrm{s}$, si la Tierra reposa en el éter cuando pasa por algún punto de su órbita, tendrá que moverse en él a unos $60 \mathrm{~km} / \mathrm{s}$ cuando pase por el punto diametralmente opuesto. Esto plantea a los físicos la tarea de medir la velocidad de la Tierra en el éter. Curiosamente, si $v$ designa la velocidad con que se mueve en el éter el aparato con que se mide la velocidad de la luz, la matemática de la teoría de la luz conlleva que se anulen los efectos ópticos de un orden de magnitud igual a $v / c .{ }^{11}$ Por tanto, para medir la velocidad de la luz con un aparato que se mueve con velocidad $v$, el equipo utilizado tiene que ser capaz de registrar efectos del orden de $v^{2} / c^{2}$. En 1879 nadie soñaba en Europa con la posibilidad de construir un aparato así y por eso Maxwell concibió un procedimiento - sofisticado y precario - para medir la velocidad de la luz observando las lunas de Júpiter que reflejaría efectos de ese orden. ${ }^{12}$ Maxwell explicó este procedimiento en una carta a D.P. Todd, de la Oficina del Almanaque Náutico de los Estados Unidos. El asunto interesó a A.A. Michelson, un joven oficial de marina asignado a dicha oficina, quien concibió y fabricó un aparato diseñado para medir tales efectos en un laboratorio terrestre, el célebre interferómetro de Michelson. Ni los primeros resultados de Michelson (1881), severamente criticados por H.A. Lorentz (1887, pp. 164ss.), ni los obtenidos con un interferómetro perfeccionado por Michelson y Morley (1887), registraron el menor movimiento de la Tierra en el éter.

El interferómetro de Michelson compara la propagación de una señal luminosa a lo largo de dos trayectos perpendiculares de la misma longitud, uno de los cuales estaría alineado con la dirección del movimiento de la Tierra en el éter mientras se realiza el experimento. Para compensar cualquier pequeña desigualdad entre los dos trayectos del aparato se los alinea alternativamente

\footnotetext{
${ }^{11}$ La anulación se produce si la Tierra arrastra consigo una pequeña fracción del éter en que está sumida. Un experimento de Fizeau (1851) había mostrado que así ocurre al parecer.

12 La propuesta de Maxwell está explicada muy claramente en A.P. French (1968), pp. 46-48.
} 
con la dirección del movimiento, haciendo girar el aparato. Pero tal compensación obviamente no tendría efecto si justamente el movimiento de un cuerpo en el éter alterase la distancia entre sus extremos en la dirección en que se mueve. H.A. Lorentz y G.F. FitzGerald propusieron independientemente esta solución al enigma planteado por el resultado negativo del experimento de Michelson y Morley. Si las partículas de un cuerpo sólido están unidas entre sí por fuerzas electromagnéticas, no sería extraño que la traslación a través del éter alterase la relación entre esas fuerzas. Sobre esta base, y sin ninguna otra indicación empírica que los respaldase, Lorentz y FitzGerald postularon que una barra de metal que se mueve en el éter con velocidad $v$ se contrae en la dirección del movimiento, multiplicándose por el factor $\left(1-v^{2} / c^{2}\right)^{1 / 2}$, que es exactamente lo que se requiere para anular los efectos ópticos del orden de $v^{2} / c^{2}$ que el movimiento de la Tierra generaría en el experimento de Michelson y Morley. Henri Poincaré ridiculizó esta hipótesis en su alocución al congreso internacional de física de 1900: "Se hicieron experimentos que habrían debido revelar los términos de primer orden; los resultados fueron negativos. ¿Sería por azar? Nadie lo admitió; se buscó una explicación general y Lorentz la encontró: mostró que los términos de primer orden debían cancelarse, pero no así los de segundo. Se hicieron entonces experimentos más precisos; también fueron negativos; tampoco esto podía ser un efecto del azar; era necesaria una explicación; se la encontró; siempre se las encuentra; las hipótesis son el recurso que menos escasea (les hypothèses, c'est le fonds qui manque le moins)" (Poincaré 1900, p. 22; 1968, p. 182). Con todo, Lorentz persistió toda su vida en su explicación de la aparente inmovilidad de la Tierra en el éter, si bien, para hacerla operante, debió suplementar su hipótesis de la contracción de las barras de medir con otra hipótesis no menos traída de los cabellos: trasladarse a través del éter retarda los relojes, alargando por un factor de $\left(1-v^{2} / c^{2}\right)^{-1 / 2}$ los intervalos de "tiempo local" medidos por un buen reloj que se mueve en el éter con velocidad $v$. (Que yo sepa, nadie ha hecho ninguna sugerencia sobre la forma cómo el campo electromagnético podría modificar precisamente de este modo la frecuencia de la radiación del cesio 133 utilizada como base para la definición de la unidad de tiempo).

En un artículo publicado en septiembre de 1905 Albert Einstein abordó de una manera radicalmente distinta el problema de la descripción de los fenómenos electromagnéticos en cuerpos que se mueven. En vez de atribuir a la materia propiedades ad hoc, calculadas específicamente para resolver las dificultades encontradas, propuso una reforma drástica de los principales conceptos empleados por la física clásica en la descripción de la naturaleza: espacio, tiempo, masa, fuerza. Para justificarla, sometió a una crítica demole- 
dora la forma tradicional de comparar el tiempo trascurrido entre eventos que ocurren en distintos lugares del espacio. Para medir la velocidad con que un cuerpo o una onda recorren la distancia entre un punto A y otro punto B, tengo que conocer esa distancia, pero también los tiempos en que el objeto sale de A y llega a B. Salvo por una breve reflexión de James Thomson, ${ }^{13}$ no hay indicios de que alguien se haya hecho cargo de la dificultad que esto último entraña antes de que Poincaré (1898) la analizara en una revista de filosofía. Al parecer, todos los físicos daban por descontado, sin más, que, como escribió Newton, "cada momento del tiempo se difunde indivisiblemente por todos los espacios" (Hall y Hall 1962, p. 104). En la $§ 1$ de su artículo de 1905, Einstein puso en evidencia la ingenuidad de esta actitud y propuso un método ideal, pero plausible y fácil de entender, para definir una coordenada temporal universal asociada a un marco inercial de referencia. No es necesario explicarlo aquí. ${ }^{14}$ Einstein demuestra que, si $(x, y, z)$ es un sistema de coordenadas cartesianas adaptado a un marco de referencia $\mathscr{H}$ que tomamos como estándar de reposo, $\left(x^{\prime}, y^{\prime}, z^{\prime}\right)$ un sistema de coordenadas cartesianas adaptado a un marco de referencia $\mathscr{H}^{\prime}$ ' que se mueve relativamente a $\mathscr{f}$ con velocidad constante $v$, y definimos en $\mathscr{\mathscr { H }} \mathrm{y} \mathscr{H}^{\prime}$, conforme al método de Einstein, las coordenadas temporales $t$ y $t^{\prime}$, respectivamente, entonces la transformación de coordenadas que lleva del sistema de coordenadas espaciotemporales $(t, x, y, z)$ al sistema $\left(t^{\prime}, x^{\prime}, y^{\prime}, z^{\prime}\right)$ es una transformación del grupo de Lorentz, esto es, precisamente el grupo de transformaciones, descubierto por Poincaré (1906, pp. 144-146), que relaciona entre sí cualesquiera dos sistemas de coordenadas espaciotemporales definidas para marcos de referencia en reposo en el éter o en movimiento uniforme respecto a él bajo los supuestos de Lorentz sobre el comportamiento de los relojes y las varas de medir en ese medio. De ello se sigue inmediatamente que toda predicción basada en los supuestos de Lorentz es también una consecuencia de los supuestos de Einstein: ambas teorías, no obstante su profunda discrepancia conceptual, son empíricamente equivalentes. ${ }^{15}$ Pero, como dice Einstein $(1905$, p. 892), su enfoque hace superflua la

\footnotetext{
13 “El tiempo requerido para la trasmisión de una señal envuelve una imperfección en las facultades humanas para determinar la simultaneidad de lo que sucede en lugares distantes. Sin embargo, no parece que envuelva ninguna dificultad para idealizar o imaginar la existencia de la simultaneidad" (Thomson 1884, p. 569).

14 Véase, por ejemplo, el artículo simultaneidad en el Diccionario de lógica y filosofía de la ciencia de Mosterín y Torretti (2010, p. 546).

15 Pablo Acuña Luongo ha estudiado este tema a fondo en su tesis doctoral (2014). Según Acuña, la principal razón para preferir la teoría de Einstein a la de Lorentz es que aquella abre el camino a la nueva teoría de la gravitación que Einstein propondrá en 1915, a la cual me referiré más adelante. En virtud de las relaciones lógicas entre ambas, la primera teoría de Einstein puede apoyarse en el abundante respaldo empírico de que goza la segunda teoría. En cambio, en un mundo acorde con esta, la teoría de Lorentz no
} 
suposición de que existe un éter portador de la luz y de las ondas electromagnéticas en general, y excluye por lo tanto cualquier efecto del movimiento en el éter sobre las varas de medir o los relojes. ${ }^{16}$ Una vez que aprendemos a ver, con Minkowski (1909), en el grupo de Lorentz el grupo característico de la geometría - o geocronometría - del espacio de cuatro dimensiones en que las coordenadas espaciotemporales representan el universo, ${ }^{17}$ la alteración de las distancias y duraciones por una transformación de Lorentz se nos revela como un efecto puramente geométrico —o geocronométrico-, análogo a las variaciones que sufren con una rotación del plano la abscisa y la ordenada cartesianas de cualquier punto distinto del origen de las coordenadas. No abundaré en las consecuencias sensacionales del cambio conceptual efectuado por Einstein. ${ }^{18}$ Menciono solo una moraleja para filósofos que me sugiere el contraste entre la postura de Lorentz y la iniciativa de Einstein: cuando la experiencia ordenada bajo ciertos conceptos impone la conclusión de que ciertas evidencias que se nos imponen son ineludiblemente ilusorias -en el ejemplo examinado, los lapsos de tiempo que miden nuestros relojes y las distancias que miden nuestras cintas métricas-, mejor que aceptar esta conclusión, sería aconsejable intentar una reforma radical de esos conceptos. Habría que tener presente esta alternativa cuando se reflexiona sobre el agnosticismo perpetuo en que, a la luz de la experiencia del cambio científico, nos sume la aceptación del concepto tradicional, aristotélico-platónico, de verdad.

En esta sección y en la próxima abordaré ejemplos de novedades empíricas inesperadas, de contenido positivo, que resulta imposible encuadrar en los sistemas conceptuales heredados e inducen por eso una innovación intelectual. ${ }^{19}$ Distingo dos tipos de casos: en el primero, la innovación intelectual sucede trabajosamente al hallazgo empírico; en el segundo, la innovación intelectual está disponible ya cuando se produce el hallazgo empírico.

\footnotetext{
tiene cabida ni siquiera a nivel local.

16 Me refiero especialmente a este último tema en Torretti (2007); versión castellana en el capítulo II de Torretti (2008).

${ }^{17}$ La idea de que cada geometría está caracterizada por un grupo fue propuesta por Felix Klein en su celebrado Programa de Erlangen (1872). Klein (1911) aplica esta idea a la teoría de Einstein (1905).

18 Lo hice en mi libro Relativity and Geometry (1983), y también, más brevemente, en castellano, en ensayos reproducidos en Torretti 1994, 2003, 2013, donde el lector hallará abundantes referencias a otras obras sobre el tema.

19 Digo de "contenido positivo" para separar estos casos del último que examinamos, la ausencia de un movimiento observable de la Tierra respecto del éter, la cual se deja describir como una novedad empírica
} 
El ejemplo señero del primer caso es, en mi opinión, el descubrimiento de la radioactividad por Henri Becquerel en febrero de 1896. Becquerel conjeturaba que la fosforencia inducida por el sol en el uranio se traducía en la emisión de rayos $\mathrm{X}$. Cuando tuvo que suspender por falta de sol un experimento con placas fotográficas destinado a probarlo, guardó en su escritorio sus sales de uranio junto a las placas protegidas con papel negro. Pocas días después decidió desarrollar las placas esperando ver registrada una imagen débil debida a la fosforescencia del uranio no asistida por el sol. Pero halló una imagen tan intensa como la que habría producido el fallido experimento. La ciencia de la época no tenía modo de explicar una radiación de esa magnitud emitida por un trozo inerte de mineral. Es dable sostener que el hallazgo de Becquerel desencadenó la serie de innovaciones conceptuales que marcan la historia de la física cuántica.

Con todo, no hay que perder de vista que Planck $(1900,1901)$ introdujo el llamado cuanto de acción - la constante $h$ que dará su nombre a la nueva física - en respuesta a una novedad menos llamativa, saber, la incongruencia entre las mediciones de la distribución de la energía en el espectro de la radiación del cuerpo negro ${ }^{20}$ efectuadas por Lunner y Pringsheim (1900) y por Rubens y Kurlbaum (1900) y los valores predichos por la "ley de desplazamiento" propuesta por Willy Wien (1893), que se aceptaba entonces como una descripción adecuada de dicha distribución.

Por otra parte, la historia de la física cuántica ha estado puntuada por una sucesión de novedades incomprensibles mediante los conceptos ya logrados, que fueron provocando una por una la referida sucesión de innovaciones. Comentaré una sola.

A comienzos del siglo XX se entendía que los cuerpos sólidos estaban llenos de materia dividida en átomos. Siguiendo a J.J.Thomson, el átomo se concebía como una esfera material positivamente cargada, regada de electrones (partículas mucho más pequeñas, con carga negativa), como un pe-

inesperada para entender la cual no había conceptos cuando se produjo a fines del siglo XIX. Pero es oportuno recordar que la expectativa de observar tal movimiento suponía, por un lado, la posibilidad de medir velocidades en diversos tiempos y lugares y respecto a distintos sistemas de referencia sin tener una definición satisfactoria de simultaneidad a distancia; y, por otro, la presencia ubicua de un cuerpo inmóvil o en movimiento inercial —el éter—que nadie jamás había visto ni tocado.

${ }^{20}$ Radiación de cuerpo negro o radiación térmica llámase la emitida por un cuerpo que absorbe toda la radiación que recibe sin reflejar ninguna parte de ella. Kirchhoff (1860) demostró que la energía irradiada en este caso no depende de la particular índole del cuerpo en cuestión sino solo de su temperatura y de la longitud de onda de la radiación. La ley de Plank expresa esta dependencia funcional. Admirablemente ratificada por experiencia, se la utiliza hoy como criterio para reconocer el carácter térmico de una radiación (por ejemplo, la radiación de fondo observada por Penzias y Wilson, de que hablaré más adelante). 
queño queque regado de pasas. Entre 1908 y 1910, experimentos realizados en el laboratorio de Ernest Rutherford por sus ayudantes Geiger y Marsden demostraron inequívocamente que este modelo del átomo era insostenible. Bombardearon con partículas $\alpha$ (esto es, átomos de helio positivamente cargados, producto de la desintegración de átomos radioactivos) una lámina de oro finísima. Si la lámina estuviera llena de materia, las partículas $\alpha$, repelidas por la carga positiva de los átomos, sufrirían una desviación inferior a un grado. Para su sorpresa y la de Rutherford, la gran mayoría de las partículas atravesaba la lámina sin cambiar de dirección, pero una parte de ellas rebotaba, con desviaciones superiores a los 90 grados. Años más tarde, Rutherford describirá así la impresión que le causó este hallazgo:

Recuerdo que ... Geiger llegó donde mí excitadísimo, diciendo: "Hemos obtenido que algunas de las partículas $\alpha$ vuelvan atrás". Fue el suceso más increíble que me hubiera ocurrido nunca. Era casi tan increíble como si dispararas una bala de cañón de 15 pulgadas contra una hoja de papel de seda y rebotara golpeándote. Reflexionando, comprendí que el rebote de cada partícula tenía que ser el efecto de una sola colisión, y cuando hice cálculos vi que era imposible obtener nada de ese orden de magnitud a menos que tomaras un sistema en que la mayor parte de la masa del átomo estuviese concentrada en un núcleo diminuto. Fue entonces que tuve la idea de un átomo con un centro masivo diminuto, portador de una carga (Rutherford 1938, pp. 68-69).

En el nuevo modelo del átomo, los electrones circulaban alrededor del núcleo como los planetas alrededor del Sol (Rutherford 1911, 1914). Sin embargo, según la electrodinámica clásica un electrón que circulara de ese modo emitiría radiación e iría perdiendo energía hasta caer en el núcleo. Esta dificultad se elimina si suponemos con Niels Bohr (1913) que los electrones del átomo no emiten radiación mientras se mueven en una órbita determinada, y solo lo hacen —en porciones (“cuantos") proporcionales a la constante $h$ de Planck - cuando saltan abruptamente de una órbita a otra. Estos "saltos cuánticos" no tenían ningún asidero en la física de entonces y resultaban ser perfectamente incomprensibles. Ello no obstante, el modelo de Bohr tuvo gran aceptación y ganó el premio Nobel de física para su inventor (en 1922), gracias a que la teoría cuantitativa que lo sustentaba permitía calcular la fórmula de Rydberg para las líneas espectrales de la radiación del hidrógeno. Los intentos de aplicarlo a átomos más complejos tropezaron con dificultades, promoviendo sucesivas correcciones de la teoría, hasta que la invención de la mecánica cuántica de matrices por Heisenberg (1925), de la mecánica ondulatoria por Schrödinger (1926), y la demostración de la equivalencia 
matemática de ambas (Schrödinger 1926, von Neumann 1927) puso fin a la era de la llamada antigua teoría cuántica e instauró una forma de hacer física dramáticamente novedosa.

Rutherford estuvo tan sorprendido por los resultados de Geiger y Marsden porque chocaban de frente con la idea intuitiva de lo que es un cuerpo sólido. ${ }^{21}$ Un fenómeno observado en su laboratorio no se dejaba pensar con arreglo a conceptos inveterados de la ciencia y del sentido común. Pero nótese bien que ese fenómeno era todo menos un "hecho crudo", una mera aglomeración de impresiones sensibles, ajena a todo concepto, como las que, en la fantasía de algunos filósofos, compondrían la materia prima de toda experiencia. Una lectura somera de los artículos de Geiger $(1908,1910)$ y de Geiger y Marsden (1909) revela que el fenómeno en cuestión, como cualquier otro fenómeno científico, se da en el contexto de una rica experiencia intelectualmente organizada, y su lectura en los términos expresados por Rutherford descansa en una multitud de inferencias y supuestos.

Finalmente, consideraré dos ejemplos del segundo caso mencionado al comienzo de la sección anterior: hechos observados que desafían la opinión recibida, pero se dejan pensar bajo un concepto ya disponible, aunque novedoso aún y desconocido para quienes los observaron por primera vez. Mis ejemplos son la fuga general de las galaxias establecida por Edwin Hubble (1929) y la radiación térmica de fondo constatada por Arno Penzias y Robert Wilson (1965), dos fenómenos descubiertos, respectivamente, más de una década y medio siglo después que Einstein (1915b) propusiera la teoría de la gravitación conocida como Teoría General de la Relatividad (TGR), ${ }^{22}$ la cual encierra conceptos apropiados para pensarlos (sin que sus descubridores lo supieran). La relación entre la TGR y los dos fenómenos aludidos suele presentarse como caso típico de la validación de una teoría científica por su ca-

21 ¿Cuántos lectores del popular libro de Eddington La naturaleza del mundo físico no han quedado perplejos ante el distingo inicial entre la mesa donde el autor escribía — “dotada de extensión, comparativamente permanente, coloreada, pero, sobre todo, sustancial"- y su "mesa científica", formada casi enteramente por un espacio vacío que recorren a gran velocidad numerosas partículas cargadas cuyo volumen conjunto asciende a menos de un mil millonésimo del que ocupa la mesa (Eddington 1929, pp. ix-x)?

22 Einstein llamó Teoría General de la Relatividad (Allgemeine Relativitätstheorie) a su teoría de la gravitación en la creencia de haría extensivo a toda clase de movimientos el Principio de Relatividad postulado en su teoría de 1905 para los movimientos inerciales (que hoy llamamos Teoría Especial de la Relatividad o TER). No es así, pero la denominación se puede justificar también de otra manera: la geocronometría minkowskiana propia de la teoría de 1905 es un caso especial de la geocronometría riemanniana característica de la TGR y esta se aproxima a aquella en un pequeño entorno de cada punto del espaciotiempo. 
pacidad de predecir fenómenos nuevos. ${ }^{23}$ Este enfoque es sin duda acertado, pero un tanto simplista, ya que, al menos en este caso, el propio Einstein no barruntó siquiera que ella implicase dichos fenómenos, y el primer indicio de que pudiera ser así provocó su abrupto — e injustificado — rechazo (Einstein 1922, comentado más adelante).

Hubble (1929) proclamó que las gran mayoría de las galaxias ${ }^{24}$ se alejan incesantemente de la Tierra con una velocidad proporcional a la distancia que nos separa de ellas. Las escasas excepciones se cuentan entre las galaxias más cercanas. Esta conclusión reposa, por una parte, en la mediciones de la velocidad radial de las galaxias realizadas por Vesto Slipher $(1913,1915,1917)$ y otros astrónomos norteamericanos, en parte en la estimación de la distancia de algunas galaxias posibilitada por el descubrimiento por Henrietta Leavitt de la relación entre la luminosidad y el período de pulsación de las estrellas llamadas Cefeidas (Pickering 1912; cf. Leavitt 1908). La medición de la velocidad radial de los astros se basaba en el efecto Doppler, en virtud del cual una onda emitida con longitud $\lambda$ desde una fuente que mueve relativamente al observador con velocidad $v$ es recibida por este con una longitud $\lambda \pm \Delta \lambda$, mayor si la fuente se aleja, menor si se acerca; donde la diferencia $\Delta \lambda$ es una

\footnotetext{
${ }^{23}$ Durante el último tercio del siglo XX, la escuela filosófica asentada en la London School of Economics sostuvo con vehemencia que el respaldo empírico de una teoría científica reposa exclusivamente en la predicción de hechos nuevos, ya que la predicción de hechos ya conocidos al tiempo en que la teoría se formula puede deberse a la inclusión en ella de hipótesis diseñadas a propósito para lograrlo (hipótesis $a d$ hoc). Como tantas otras idées fixes de las escuelas, esta no calza con la práctica científica ni con el sentido común. Antes bien, lo normal es que el pensamiento científico se alimente de la reflexión sobre hechos conocidos que no sabemos explicar ni predecir hasta dar con una idea que nos permita entenderlos $\mathrm{y}$, con ello, preverlos.

${ }^{24}$ En su artículo, Hubble llama 'nebulosas extragalácticas' (extra-galactic nebulae) a los objetos astronómicos que hoy llamamos galaxias; 'extragaláctico' significa que se encuentra fuera de la Vía Láctea, el grupo de 100.000.000.000 de estrellas al que pertenece el Sol que es la galaxia por antonomasia ( $\gamma \alpha \lambda \alpha \xi^{\prime} \alpha_{\alpha \varsigma}$ en griego, derivado de $\gamma \alpha \dot{\lambda} \lambda \alpha$, 'leche'). Este nombre se hizo extensivo a las nebulosas extragaláctica en cuanto la comunidad científica se convenció de que estos objetos son grupos de estrellas análogos a la Vía Láctea, situados a gran distancia de esta. Aunque Herschel, el descubridor de Urano, y el filósofo Kant sostuvieron esto en el siglo XVIII, hasta la década de 1920 prevaleció la tesis de que todas las nebulosas observables en el cielo son masas gaseosas, situadas dentro de la Vía Láctea, que constituyen criaderos de estrellas. La tesis de Herschel y Kant se impuso finalmente gracias a las mediciones de velocidad, iniciadas por Slipher hacia 1910, a que me refiero en el texto. Según Lang y Gingerich (1979, p. 713) "el gran debate sobre la naturaleza extragaláctica de las nebulosas espirales" quedó resuelto finalmente cuando la AAS y la AAAS, reunidas conjuntamente en Washington, tomaron conocimiento el $1^{\circ}$ de enero de 1925 del trabajo de Hubble "Cefeidas en nebulosas espirales" (1925). En todo caso, el Oxford English Dictionary cita textos de 1848 y 1888 donde la voz galaxy se emplea en su acepción actual; el segundo expresa claramente el tránsito de una acepción a la otra: " 1888 C. A. Young Gen. Astron. xxi. 503: The belief that these star-clusters are stellar universes,- ' 'galaxies', like the group of stars to which the writers supposed the sun to belong".
} 
función de $v \cdot{ }^{25} \mathrm{El}$ cambio de longitud de onda de la luz procedente de una estrella se mide comparando la posición que tienen en dicha luz las rayas espectrales características de los elementos químicos presentes en esa estrella con la posición de tales rayas en la radiación emitida por los mismos elementos en un laboratorio terrestre. El aumento de la longitud de onda se traduce en un desplazamiento de todas las rayas hacia el rojo; su disminución en un corrimiento hacia el azul. Las referidas mediciones astronómicas revelaron muy pronto que, mientras que el espectro de las estrellas más cercanas se desplaza indiferentemente hacia el azul o hacia el rojo, el de las galaxias mayormente lo hace hacia el rojo. Slipher (1915) informa sobre la velocidad radial de 15 galaxias, de las cuales 3 se acercan con una velocidad de unos $300 \mathrm{~km} / \mathrm{s}, 9$ se alejan con velocidades entre 200 y $110 \mathrm{~km} / \mathrm{s}$, y 3 lo hacen con velocidades pequeñas (una de estas quizás se acerque). Slipher (1917) informa sobre 25 (con valores revisados para las 15 anteriores), de las cuales 4 se acercan con velocidades entre 30 y $360 \mathrm{~km} / \mathrm{s}$ y 21 se alejan con velocidades entre 150 y $1100 \mathrm{~km} / \mathrm{s}$. Ocho años más tarde, Strömberg (1925) enumera 57 galaxias observadas por Slipher, y 5 más reportadas por otros observadores; 48 se alejan con velocidades entre 10 y $1800 \mathrm{~km} / \mathrm{s}$ y solo 13 se acercan con velocidades entre 10 y $300 \mathrm{~km} / \mathrm{s}$. Obviamente, para derivar estas velocidades de la magnitud del desplazamiento de las líneas espectrales hay que suponer que estas ocupan en la luz emitida hoy en laboratorios terrestres la misma posición que ocupaban hace muchos años en la que emitieron las galaxias estudiadas, las cuales se hallan a distancias enormes de nosotros. No nos cabe obrar de otro modo si cumplimos con la $4^{\text {a }}$ regla de filosofar de Newton (1726, p. 389): "En la filosofía experimental, las proposiciones recogidas de los fenómenos por inducción deben ser tenidas por verdaderas exactamente o tan aproximadamente como sea posible, no obstante las hipótesis contrarias, hasta que ocurran otros fenómenos, que las hagan más exactas o les opongan excepciones". Razonar bajo esta regla expone sin duda a la ciencia al riesgo de tener que retractarse a la luz de fenómenos nuevos, pero renunciar a ella la paralizaría, condenándola a una perpetua y estéril suspensión del juicio.

Dos años antes del anuncio de Hubble sobre la proporcionalidad entre las velocidades de fuga y las distancias, el astrónomo belga Georges Lemaître (1927) había llamado mucho la atención con una propuesta basada en la

\footnotetext{
${ }^{25}$ El efecto es familiar: cuando una ambulancia pasa rápidamente a nuestro lado y, dejando de acercársenos, procede a alejarse, el sonido de la sirena cambia bruscamente de agudo a grave. La relación de dependencia entre $\Delta \lambda$ y $\mathrm{v}$ se expresa en la física clásica mediante una fórmula que es una aproximación de primer orden (v/c) a la fórmula propia de la TER (nota 22). Como la fórmula de la TER se deduce de las transformaciones de Lorentz, el efecto Doppler resulta ser un efecto geocronométrico.
} 
TGR, que explica el fenómeno como una manifestación de la geometría del universo. La explicación de Lemaître, que describiré luego, es esencialmente la misma que se acepta hoy, pero no fue el primer intento de conectar la TGR y la fuga de las galaxias. Para motivarla mejor, narro sumariamente esta historia.

La TGR se resume en un sistema de ecuaciones diferenciales, las ecuaciones de campo de Einstein (ECE), publicado el 27 de noviembre de 1915 (Einstein 1915b). La primera solución exacta de estas ecuaciones, debida a Karl Schwarzschild, fue presentada a la Academia de Berlín el 13 de enero de 1916. Ella ofrece un modelo idealizado del movimiento de una partícula de prueba (esto es, de masa tan pequeña que no afecte el campo gravitacional) en un campo gravitacional estático y esféricamente simétrico. Esta solución, que permitió calcular con notable exactitud la precesión secular del perihelio de Mercurio, no explicada por la teoría de la gravedad de Newton, ${ }^{26}$ juega hoy un rol decisivo en la vida diaria de cualquiera que utilice el GPS. Como condición de frontera para su solución, Schwarzschild supone que la métrica del espaciotiempo converge en el infinito a la métrica de Minkowski. ${ }^{27}$ De este modo, a juicio de Einstein, ella reintroduce el sistema inercial ("ese fantasma que actúa sobre todo, sin que las cosas actúen de vuelta sobre él" - Einstein a Born, 12.05.1952; en Einstein, Born y Born 1969, p. 258), en cuya eliminación él veía uno de los mayores logros de la TGR. ${ }^{28}$ Einstein (1917) presenta una solución exacta de las ECE sin condiciones de frontera, que brinda en efecto un modelo del universo, el primer modelo cosmológico de la TGR. Se trata de un universo estático en que el espacio tiene una geometría riemanniana de curvatura positiva constante y, por lo tanto, es ilimitado pero finito (piénsese en la superficie de una esfera, el análogo bidimensional del espacio de este modelo). Para asegurar el carácter estático del universo, Einstein tuvo que agregar un término nuevo a las ECE de 1915, consistente en el tensor métrico $g_{i j}$ multiplicado por una constante arbitraria $\Lambda \neq 0 .{ }^{29}$ En el modelo estático

\footnotetext{
${ }^{26}$ De hecho, Einstein (1915a) ya había calculado la anomalía de Mercurio utilizando una solución aproximada de las ECE.

${ }^{27}$ George Birkhoff (1923) demostrará luego que este supuesto es innecesario: la convergencia a la métrica de Minkowski se deduce de la suposición de que el campo gravitacional es esféricamente simétrico.

${ }^{28}$ En el espaciotiempo de Minkowski, la geocronometría prescribe las trayectorias de las partículas libres, pero no es afectada por la presencia de estas.

${ }^{29}$ La constante $\Lambda$, conocida en la literatura como la constante cosmológica ha tenido una historia accidentada. Cuando quedó establecido que el universo real no es estático, Einstein lamentó haberla introducido y derivó una solución de las ECE correspondiente a un universo que se expande, poniendo $\Lambda=0$ (Einstein y de Sitter 1932). Pero desde que se estableció a fines del siglo XX que la expansión del universo es acelerada (Hamuy et al. 1996, Schmidt at al. 1998, Perlmutter et al. 1998), la constante $\Lambda$ se identifica con la
} 
propuesto, $\Lambda$ es inversamente proporcional al cuadrado del radio del universo esférico, y este cuadrado a su vez es proporcional a la densidad media de la materia. En apoyo de su modelo, Einstein — que obviamente no conocía los hallazgos de Slipher - señala que una distribución aproximadamente estática de la materia "refleja el hecho de que las velocidades estelares son pequeñas" (der Tatsache der kleinen Sterngeschwindigkeiten entspricht-Einstein 1917, p. 152). Por su parte, en un artículo publicado en Inglaterra ese mismo año, el astrónomo holandés Willem de Sitter, que algo sabía de esos hallazgos, los invoca en pro del modelo cosmológico alternativo propuesto por él: un universo vacío, topológicamente equivalente a una esfera de cuatro dimensiones, en que las partículas de prueba en caída libre trazan cosmolíneas ${ }^{30}$ geodésicas divergentes. De Sitter solo aduce mediciones de la velocidad radial de tres galaxias. "Sin embargo - escribe-, si la observación continua confirmase el hecho de que las nebulosas espirales sistemáticamente exhiben velocidades radiales positivas, esto sería una indicación para adoptar la hipótesis B [la suya], con preferencia a la A [de Einstein]" (de Sitter 1917, p. 28). Strömberg (1925, p. 360) señala que la correlación observada entre distancia y velocidad de alejamiento respalda el modelo de de Sitter. ${ }^{31}$

En el §1 de su artículo, Lemaître (1927) enfrenta los modelos cosmológicos de Einstein y de Sitter. Ambos, dice, tienen sus ventajas: el segundo "concuerda con la observación de las velocidades radiales de las nebulosas", mientras que el primero "tiene en cuenta la presencia de materia y da una relación satisfactoria entre el radio del universo y la masa que contiene" ( $p$. 50). Sería deseable, pues, obtener una solución intermedia que reúna ambas ventajas, pero tal solución es aparentemente imposible. En efecto, las ECE no admiten sino estas dos soluciones para un campo gravitacional estático y de simetría esférica, si la materia está uniformemente distribuida y no está sometida a presiones o tensiones internas. Por otra parte, la impresión de que el universo de de Sitter es estático resulta de la adopción de coordenadas que "introducen un centro al que nada corresponde en la realidad" y dividen

\footnotetext{
densidad de la energía del vacío y se le asigna un valor superior a 0.

${ }^{30}$ Cf. Mosterín y Torretti (2010, p. 143): “cosmolínea (A. Weltlinie, F. ligne d'univers, I. worldline). Los eventos sucesivos de la historia de una partícula forman el trayecto o camino de una curva en el espaciotiempo. Esta curva, parametrizada por el tiempo propio, es la cosmolínea de la partícula. Cuando se habla de la cosmolínea de un cuerpo, esto puede referirse o bien a la cosmolínea de su centro de masa, o bien - con cierta impropiedad - a la región del espaciotiempo que ocupan los eventos de la historia de ese cuerpo, y que sería tal vez más apropiado llamar su cosmotubo".

31 También Hubble (1929, p. 173) alude a ello, diciendo que "el rasgo más sobresaliente... es la posibilidad de que la relación entre velocidad y distancia represente el efecto de Sitter y que, por tanto, se introduzcan datos cuantitativos en los debates sobre la curvatura general del espacio".
} 
el espaciotiempo en espacio y tiempo de una manera que no respeta la homogeneidad de aquel (ibid.). "Si se introducen coordenadas y una división correspondiente del espacio y el tiempo que respeten la homogeneidad del universo, se comprueba que el campo no es estático y se obtiene un universo de la misma forma que el de Einstein, pero donde el radio del espacio no permanece invariable sino que varía con el tiempo" (p. 51). Motivado por estas consideraciones, Lemaître emprende la búsqueda de una solución de las ECE que corresponda a un universo con espacio esférico de radio variable. Supone que la materia es un fluido perfecto (densidad uniforme, presión isotrópica constante en todo lugar) y demuestra que bajo esta condición idealizante se obtiene un modelo cosmológico que cumple las condiciones siguientes (p. 58): $1^{\circ}$ La masa $\mathrm{M}$ del universo es constante y es proporcional a $2 \pi^{2} \Lambda^{-1 / 2} \cdot 2^{\circ}$ El radio del universo crece sin cesar desde un valor asintótico $\mathrm{R}_{0}$ para $t=-\infty .3^{\circ} \mathrm{La}$ fuga de las nebulosas extragalácticas se debe a la expansión del espacio mismo ${ }^{32}$ y permite calcular el radio $\mathrm{R}_{0} .4^{\circ} \mathrm{El}$ radio actual del universo depende de la densidad actual de la materia, según una ecuación similar a la que relaciona radio y densidad en el universo estático de Einstein. Este es, en efecto, un caso límite de universo de Lemaître y, como tal, es inherentemente inestable, es decir, pierde el carácter estático a la menor variación en la densidad de la materia (Eddington 1930). ${ }^{33}$

El artículo de Lemaître fue el vehículo que trajo a la comunidad astronómica la explicación relativista de la fuga de las galaxias como consecuencia de la expansión del universo. Pero la idea misma de una cosmología dinámica ya había sido presentada antes, de una manera más clara y elegante, por el matemático y meteorólogo ruso Alexander Friedmann $(1922,1924)$ en dos artículos publicados en la Zeitschrift für Physik. Friedmann no hace la menor alusión al fenómeno observado por los astrónomos americanos, sino que procede simplemente a la derivación matemática de una familia de soluciones exactas de las ECE, a la cual pertenecen, como casos límite, las soluciones de

\footnotetext{
32 En otras palabras, el corrimiento hacia el rojo de las líneas espectrales en la luz proveniente de las galaxias no se debe, como supusieron Slipher y Hubble, a la velocidad con que las galaxias se mueven relativamente a nosotros en el espacio eterno, sino a que, mientras la luz viajaba entre la galaxia y nosotros, el espacio mismo se ha dilatado, con lo cual ha ido creciendo la distancia entre las crestas consecutivas de las ondas de luz. Ello permite dar cuenta inmediatamente de la proporcionalidad entre corrimiento al rojo y distancia, puesto que, debido a la constancia de la velocidad de la luz, la distancia que la luz que recorre entre una galaxia y la Tierra es proporcional al tiempo que demora en recorrerla. Adviértase cómo en este caso la explicación teórica del fenómeno rectifica el concepto bajo el cual los observadores inicialmente lo entendían.

33 Por esta razón, el universo estático de Einstein es inadmisible como hipótesis científica, ya que en la ciencias empíricas las cantidades continuas no pueden determinarse exactamente, sino solo dentro de un margen finito de error.
} 
Einstein (1917) y de Sitter (1917). No abundaré en ello aquí. ${ }^{34}$ Básteme señalar que Friedmann, como Lemaître, supone que la materia está distribuida en todo el espacio, pero en aras de la simplicidad, postula que consiste en polvo inconexo (Lemaître en cambio supone que es un fluido perfecto, una condición un poco más realista que la anterior). Bajo este supuesto, genera soluciones correspondientes a varias clases de modelos cosmológicos. Algunos universos de Friedmann se expanden indefinidamente a partir de un instante pretérito separado del presente por un lapso finito de tiempo, en el cual, si el universo hubiera existido en ese instante, la densidad de la materia superaría cualquier magnitud asignable. Otros se contraen desde toda la eternidad hasta un instante futuro separado del presente por un lapso finito de tiempo, en el cual, si el universo existiere en ese instante, la densidad de la materia superaría cualquier magnitud asignable. Otros, por último, se expanden durante un lapso de tiempo finito como los universos de la primera clase, para luego contraerse durante un lapso de tiempo finito como los de la segunda. En los universos de esta última clase el espacio tiene curvatura positiva uniforme como en el universo estático de Einstein, pero ella disminuye continuamente en la primera fase y aumenta continuamente en la segunda. Los universos de las otras dos clases tienen espacios de curvatura uniforme negativa, que varía con el tiempo, o es siempre igual a cero y por tanto constante. ${ }^{35}$

Como ya anuncié, mi último ejemplo concierne a la explicación relativista de la radiación térmica de fondo. Penzias y Wilson la descubrieron durante la fase preparatoria de un proyecto de investigación radiotelescópica en que se estaban embarcando. Emprendieron mediciones para calibrar bien el equipo utilizado y maximizar su precisión. La pieza principal del mismo era una antena reflectora con forma de cuerno de 20 pies de diámetro, diseñada para captar de preferencia las señales provenientes de la dirección en que apuntara la antena y excluir las provenientes del suelo. Penzias y Wilson quisieron "hacer mediciones exactas de las temperaturas de la antena" (Wilson 1978, p. 467). Desde un comienzo les sorprendió registrar en todas direcciones una temperatura total de unos 7,5 K, cuando no esperaban más de 3,3 K (2,3 K procedente de la atmósfera, $1 \mathrm{~K}$ del suelo y las paredes de la antena). En vano buscaron una fuente del exceso, pues este se manifestaba en cualquier dirección que apuntasen la antena. La única alternativa admisible era que el exceso procediera del aparato mismo. Calcularon que este contribuiría $0.9 \mathrm{~K}$

\footnotetext{
${ }^{34}$ Lo hice en el capítulo 5 de Torretti 2003.

35 Después de la aparición del primer artículo de Friedmann, la Zeitschrift für Physik publicó una brevísima nota de Einstein (1922), en la que este dice que aquel reposa en un error matemático. Al año siguiente, en otra nota muy breve, Einstein (1923) se retractó.
} 
por calentamiento resistivo (efecto Joule). Una pequeña reducción adicional de la temperatura de la antena se obtuvo cuando desalojaron a dos palomas que anidaban en ella y lavaron sus excrementos. Durante casi un año hicieron caso omiso del problema, que persistió sin cambios, a pesar de que al cabo de ese tiempo cualquier fuente situada dentro del sistema solar habría cambiado mucho de dirección, lo cual debería afectar la temperatura de la antena. El enigma solo pudo descifrarse después que Penzias, en una conversación casual con un colega de M.I.T., se enteró de que en Princeton, R.H. Dicke y su grupo estudiaban la radiación cósmica emitida durante la fase inicial, sumamente densa y caliente, de un universo en expansión. Entraron en contacto con ellos y finalmente acordaron publicar dos cartas en el Astrophysical Journal, una sobre la teoría desarrollada en Princeton (Dicke et al. 1965), otra sobre la medición de un exceso de temperatura en la antena de los Laboratorios Bell (Penzias y Wilson 1965).

La carta de Dicke y sus asociados va por delante. En ella, adoptan tentativamente como hipótesis de trabajo una interpretación de la cosmología relativista que gozó de bastante favor justamente hasta $1965 .{ }^{36}$ Ella da por descontado que el modelo cosmológico que mejor se ajusta a la realidad es el universo de Friedmann que sucesivamente se expande y se contrae, y supone que la singularidad que este modelo sitúa al comienzo de la expansión del universo es una representación idealizada del tránsito entre una fase anterior de contracción y la actual fase expansiva, durante el cual la densidad y temperatura de la materia alcanzarían valores elevadísimos, pero sin exceder toda magnitud asignable como implica el razonamiento de Friedmann. Bajo esta hipótesis, es forzoso admitir "que en el momento de máximo colapso la temperatura del universo sería superior a $10^{10} \mathrm{~K}$," para que las cenizas de la fase anterior se conviertan nuevamente en el hidrógeno necesario para las estrellas de la nueva fase (Dicke et al. 1965, p. 415). "Pero aunque el universo tuviera un origen singular hubiera sido extremadamente caliente en sus etapas más tempranas" (Ibid.). En ambos casos cabe esperar que subsistan hasta hoy vestigios de la radiación térmica generada por esa bola de fuego, enfriados por la expansión del universo. Peebles y Wilkinson (que con Dicke y Roll firman la carta) habían iniciado un investigación encaminada a hallar esos vestigios cuando se enteraron de que Penzias y Wilson habían observado radiación

\footnotetext{
${ }^{36}$ Dicha interpretación trataba la singularidad (o "agujero blanco") inicial del universo en expansión como mero artefacto matemático. En trabajos publicados a partir de ese año, Penrose (1965), Hawking (1965, 1966, 1966/67), Geroch (1966) y Hawking y Penrose (1970) demostraron que es prácticamente inevitable que un espaciotiempo relativista exhiba singularidades. El artículo singularidad de Mosterín y Torretti (2010) explica someramente este concepto.
} 
de fondo con longitud de onda de 0,073 m. Descontadas todas las fuentes conocidas de la radiación observada, quedaba sin explicar una temperatura de $3,5 \pm 1 \mathrm{~K}$.

Para identificar la inexplicable radiación isotrópica registrada en la antena de los Laboratorios Bell con la radiación cósmica de fondo buscada por el grupo de Princeton era indispensable efectuar mediciones a muchas otras longitudes de onda, pues solo conociendo el espectro completo podría determinarse si era o no radiación térmica (cf. la nota 19). Debido a que la atmósfera opaca las señales de radio de longitud inferior a $0,03 \mathrm{~m}$ (lo que incluye precisamente aquellas a las que corresponde la máxima intensidad de una radiación térmica a la temperatura indicada, y por tanto el característico punto de inflexión de la curva predicha por la Ley de Planck), esta comprobación solo pudo completarse en la década de 1990, gracias a las observaciones registradas con el satélite COBE lanzado en 1989 con este propósito. Pero estas observaciones confirmaron todas las expectativas con una precisión realmente abrumadora. ${ }^{37}$

En su carta paralela, Penzias y Wilkins se limitan a explicar el equipo utilizado y los resultados obtenidos, omitiendo toda interpretación cosmológica, aunque agradecen expresamente a Dicke y sus socios "la fecunda discusión de estos resultados, previa a su publicación" (1965, p. 420). En una "nota agregada a las pruebas" mencionan una medición anterior de la radiación del fondo del cielo, la cual, "combinada con nuestro resultado... elimina la posibilidad de que la radiación que observamos se deba a fuentes emisoras de un tipo conocido, pues en tal caso el espectro sería mucho más empinado" (p. 421). No hacen la menor alusión a que pudiera tratarse de radiación térmica. En la conferencia que Wilson dictó en 1978, cuando le otorgaron el premio Nobel, dedica la sección XII a este asunto. Menciona múltiples mediciones efectuadas después de 1965 a gran altura, aunque dentro de la atmósfera, las cuales "establecen que la radiación de fondo tiene un espectro de cuerpo negro que sería muy difícil de reproducir con una fuente cósmica de otro tipo; la fuente tiene que haber sido ópticamente gruesa y por tanto tiene que haber existido antes que cualquier otra de las fuentes que se pueden observar"

\footnotetext{
${ }^{37}$ Recomiendo vivamente examinar la gráfica de intensidad vs. longitud de onda de la radiación de fondo que figura el $\mathrm{n}^{\circ} 3$ del artículo "¿Por qué me creo... el Big Bang?” publicado por José Luis Blanco en http://www.ciencia-explicada.com/2009/08/por-que-me-creo-el-big-bang.html. Téngase en cuenta que los intervalos de error indicados en esta gráfica han sido multiplicados por 400 para hacerlos visibles sobre el papel.
} 
(Wilson 1978, p. 482). Reproduce una gráfica de las mediciones, la cuales no se extienden más allá de la cúspide del espectro, y en la vecindad de esta son mucho menos precisas que las mediciones del COBE.

La doble publicación de Dicke et al. y de Penzias y Wilson en el Astrophysical Journal de 1965 parece calculada para ilustrar la tradicional dualidad filosófica de razón y empirie, conceptos y datos. Por un lado el fenómeno inesperado y desconcertante; por otro la teoría preexistente, desconocida para los descubridores del mismo, presta para dar cuenta de él. Pero basta leer atenta y reflexivamente la extensa y luminosa conferencia Nobel de Wilson para convencerse de que también la llamada empirie está empapada de razón y de que el encuentro con la teoría reconstituye el fenómeno. La descripción del complejo equipo utilizado despliega un arsenal de conceptos sin los cuales es imposible apreciar el significado y la relevancia de los números registrados en las observaciones. Por su parte, la aplicación de la teoría reconcibe el fenómeno, y lo integra en nuevos procesos de búsqueda inteligente, como el que llevó en este caso a reconstruir el espectro completo de la radiación de fondo, ajustado a la ley de Planck.

\section{Referencias bibliográficas}

AcuÑa Luongo, Pablo (2014). Empirical Equivalence and Underdetermination of Theory Choice: A Philosophical Appraisal and Two Case-Studies. Utrecht University. Institute for History and Foundations of Science. Ph.D. thesis.

Benveniste, Émile (1969). Le vocabulaire des institutions indo-européennes. 1.Économie, parenté, société. 2. Pouvoir, droit, religion. Paris: Éditions de Minuit. 2 vols.

BiRKhoff, George David (1923). Relativity and Modern Physics. Cambridge, MA: Harvard University Press.

BoHr, Niels (1913). "On the Constitution of Atoms and Molecules". Philosophical Magazine 26: 1-24, 476-502, 857-875.

Buchwald, Jed Z. (1995). The Creation of Scientific Effects: Heinrich Hertz and Electric Waves. Chicago: University of Chicago Press.

Chapin, Seymour L. (1985). “A Legendary Bon Mot?: Franklin's «What Is The Good of a Newborn Baby?»." Proceedings of the American Philosophical Society. 129: 278-290. 
Cohen, I. Bernard (1987). "Faraday and Franklin's «Newborn Baby»". Proceedings of the American Philosophical Society. 131: 177-182.

Coulomb, Charles-Augustin de (1784). "Recherches théoriques et expérimentales sur la force de torsion et sur l'élasticité des fils de metal," Histoire de l'Académie Royale des Sciences, pp. 229-269.

Dicke, R. H., et al. (1965). "Cosmic black-body radiation". Astrophysical Journal. 142: 414-419.

Eddington, Arthur S. (1930). "On the instability of Einstein's spherical world". Monthly Notices of the Royal Astronomical Society. 90: 668678.

EINSTEIN, Albert (1905). "Zur Elektrodynamik bewegter Körper”. Annalen der Physik. (4) 17: 891-921.

EInsteIn, Albert (1915). "Die Feldgleichungen der Gravitation". K. Preussische Akademie der Wissenschaften. Sitzungsberichte. 1915: 844-847.

EINSTEIN, Albert (1917). "Kosmologische Betrachtungen zur allgemeinen Relativitätstheorie". Preussische Akademie der Wissenschaften. Sitzungsberichte. 1917: 142-152.

Einstein, Albert (1922). "Bemerkung zu der Arbeit von A. Friedmann, 'Über die Krümmung des Raumes". Zeitschrift für Physik. 11: 326.

EInSteIn, Albert (1923). "Notiz zu der Arbeit von A. Friedmann, 'Über die Krümmung des Raumes"'. Zeitschrift für Physik. 16: 228.

Einstein, Albert \& DE SitTer, Willem (1932). "On the relation between the expansion and the mean density of the universe". Proceedings of the National Academy of Sciences. 18: 213-214.

Einstein, Albert \& Born, Hanna \& Born, Max (1969). Briefwechsel 19161955. München: Nymphenburgerger Verlagshandlung.

Fizeau, A. H. L. (1851). "Sur les hypothèses relatives à l'éther lumineux, et sur une expérience qui parait démontrer que le mouvement des corps change la vitesse avec laquelle la lumière se propage dans leur intérieur". Comptes Rendus de l'Académie des Sciences. 33: 349-355.

French, A.P. (1968). Special Relativity. New York: Norton.

FriedmanN, Alexander (1922). "Über die Krümmung des Raumes". Zeitschrift für Physik. 10: 377-386. 
Friedmann, Alexander (1924). "Über die Möglichkeit einer Welt mit konstanter negativer Krümmung des Raumes". Zeitschrift für Physik. 21: 326-332.

GaliLeI, Galileo (1638). Discorsi e dimostrazioni matematiche intorno à due nuove scienze attenenti alla Mecanica \& $i$ Movimenti Locali. Leida: appresso gli Elsevirii.

Galilei, Galileo (1964). Le opere. Firenze: G. Barbera.

Geiger, Hans (1908). "On the Scattering of $\alpha$-Particles by Matter". Proceedings of the Royal Society of London. A 81: 174-177.

GeIGER, Hans (1910). “The Scattering of the $\alpha$-Particles by Matter". Proceedings of the Royal Society of London. A 83: 492-504.

Geiger, Hans \& Marsden, Ernest (1909). "On a Diffuse Reflection of the $\alpha$-Particles" Proceedings of the Royal Society of London. A82: 495500 .

Geroch, R. P. (1966). "Singularities in closed universes". Physical Review Letters. 17: 445-447.

Hall, A. Rupert \& Hall, Marie Boas (1962). Unpublished Scientific Papers of Isaac Newton. Cambridge: Cambridge University Press.

Hamuy, Mario et al. (1996). "The Absolute Luminosities of the Calan/Tololo Type Ia Supernovae”. Astronomical Journal. 112: 2391-2397.

Hawking, Stephen W. (1965). "Occurrence of singularities in open universes". Physical Review Letters. 15: 689-690.

Hawking, Stephen W. (1966). "Singularities in the universe". Physical Review Letters. 17: 444-445.

HawkING, Stephen W. (1966/67). "The occurrence of singularities in cosmology”. Royal Society of London Proceedings. A 294: 511-521; A 295: 490493; A 300: 187-201.

Hawking, Stephen W. \& Penrose, Roger (1970). "The singularities of gravitational collapse and cosmology". Royal Society of London Proceedings. A 314: 529-548.

HeISENBERG, Werner (1925). "Über quantentheoretische Umdeutung kinematischer und mechanischer Beziehungen". Zeitschrift für Physik. 33: 879-893. 
Hertz, Heinrich (1887). "Ueber sehr schnelle elektrische Schwingungen". Annalen der Physik. 31: 421-448.

HeRTZ, Heinrich(1892). Untersuchungen ueber die Ausbreitung der elektrischen Kraft. Leipzig: Barth.

HERTz, Heinrich (1893). Electric Waves, being researches on the propagation of electric action with finite velocity through space. Authorized English translation by D.E. Jones, with a Preface by Lord Kelvin. London: Macmillan. (Reeditado por Dover Publications).

Hubble, Edwin P. (1925). "Cepheids in spiral nebulae". Publications of the American Astronomical Society. 48: 139-142.

Hubble, Edwin P. (1926). "Extra-Galactic Nebulae". Astrophysical Journal. 64: 321-369.

HubBLE, Edwin P. (1929). "A relation between distance and radial velocity among extragalactic nebulae". Proceedings of the National Academy of Sciences. 15: 168-173.

Hubble, Edwin P. \& Humason, M. L. (1931). "The velocity-distance relation among extra-galactic nebulae”. Astrophysical Journal. 74: 43-80.

KLEIN, Felix (1872). Vergleichende Betrachtungen über neuere geometrische Forschungen. Erlangen: A. Duchert.

KLEIN, Felix (1911). “Über die geometrischen Grundlagen der Lorentz-Gruppe”. Physikalische Zeitschrift. 12: 17-27.

Leavitt, Henrietta (1908). "1777 Variables in the Magellanic Clouds". Annals of Harvard College Observatory. 70: (4) 87-108.

LEMAître, Georges (1927). "Un univers homogène de masse constante et de rayon croissant, rendant compte de la vitesse radiales des nébuleuses extra-galactiques". Annales de la Société des Sciences de Bruxelles. A 47: 49-59.

Lorentz, H.A. (1887). "De l'influence du mouvement de la terre sur les phénomènes lumineux". Archives néerlandaises des sciences exactes et naturelles. 21 (1887): 103-176. [Traducción francesa de H.A. Lorentz, "Over den invloed, dien de beweging der aarde op de lichtverschijnselen uitoefent". Koninklijke Akademie van Wetenschappen (Amsterdam). Afdeeling Natuurkunde. Verslagen en Mededeelingen. 2 (1885-86): 297-372.] 
Lummer, Otto \& PringsheIM, Ernst (1900). "Über die Strahlung des schwarzen Körpers für lange Wellen”. Verhandlungen der deutschen physikalischen Gesellschaft. 2: 163-180.

Maxwell, James Clerk (1861/62). "On physical lines of force”. Philosophical Magazine. 21: 161-175, 281-291, 338-345 (1861); 23: 12-24, 85-95 (1862).

Maxwell, James Clerk (1864). "A dynamical theory of the electromagnetic field". Royal Society of London Philosophical Transactions. 155: 459512.

Michelson, A. A. (1881). "The relative motion of the earth and the luminiferous ether”. American Journal of Science. 22: 120-129.

Michelson, A. A. \& Morley, E. W. (1887). "On the relative motion of the earth and the luminiferous aether". Philosophical Magazine. (5) 24: 449-463.

Minkowski, Hermann (1909). "Raum und Zeit". Physikalische Zeitschrift. 10: 104-111.

Mosterín, Jesús \& Torretti, Roberto (2010). Diccionario de lógica y filosofía de la ciencia. $2^{\text {a }}$ edición. Madrid: Alianza Editorial. [La primera edición fue publicada por Alianza Editorial en 2002].

Newton, Isaac (1687). Philosophice naturalis principia mathematica. Londini: Jussu Societatis Regiae ac Typis Josephi Streater.

Newton, Isaac (1726). Philosophice naturalis principia mathematica. Editio tertia aucta \& emendata. Londini: Apud Guil. \& Joh. Innys, Regiæ Societatis typographos.

Onians, Richard Broxton (1951). The Origins of European Thought about the Body, the Mind, the Soul, the World, Time and Fate. Cambridge: Cambridge University Press.

Penrose, Roger (1965). "Gravitational collapse and space-time singularities". Physical Review Letters. 14: 57-59.

Penzias, Arno A. \& Wilson, Robert W. (1965). "A measurement of excess antenna temperature at $4080 \mathrm{MHz}$ ". Astrophysical Journal. 142: 419421.

Perlmutter, S. et al. (1998). "Discovery of a supernova explosion at half the age of the Universe". Nature. 391: 51-54. 
Pickering, Edward C. (1912). "Periods of 25 variable stars in the small Magellanic cloud". Harvard College Observatory Circular 173. pp. 1-3.

Planck, Max (1900). "Zur Theorie des Gesetzes der Energieverteilung im Normalspectrum". Verhandlungen der deutschen physikalischen Gesellschaft. 2: 237-245.

Planck, Max (1901). "Über das Gesetz der Energieverteilung im Normalspectrum". Annalen der Physik, 4: 553-563.

PoInCARÉ, Henri (1898). "La mésure du temps". Revue de métaphysique et de morale. 6: 1-13.

PoInCARé, Henri (1900). Sur les rapports de la physique expérimentale et de la physique mathématique. Rapport présenté au Congrès International de Physique, réuni à Paris en 1900, sous les auspices de la Société Française de Physique. Paris: Gauthier-Villars, 1900. (Reproducido en Poincaré 1968: 158-190).

Poincaré, Henri (1906). "Sur la dynamique de l'électron". Rendiconti del Circolo matematico di Palermo. 21: 129-175.

PoInCARÉ, Henri (1968). La science et l'hypothèse. Paris: Flammarion.

Pope, Alexander (1963). The Poems. A one-volume edition of the Twickenham text with selected annotations, edited by John Butt. London: Methuen \& Co.

Rubens, Heinrich \& Kurlbaum, F. (1900). "Úber die Emission langwelliger Wärmestrahlen durch den schwarzen Körper bei verschieden Temperaturen". Preussische Akademie der Wissenschaften. Sitzungsberichte. pp. 929-941.

Rutherford, Ernest (1911). "The Scattering of $\alpha$ and $\beta$ Particles by Matter and the Structure of the Atom". Philosophical Magazine. (6) 21: 669-688.

Rutherford, Ernest (1914). "The Structure of the Atom". Philosophical Magazine. (6) 27 (159): 488-498.

Schmidt, B.F. et al. (1998). "The High-Z Supernova Search: Measuring Cosmic Deceleration and Global Curvature of the Universe Using Type Ia Supernovae". Astrophysical Journal. 507: 46-63.

SCHRÖDINGER, Erwin (1926a). "Quantisierung als Eigenwertproblem”. Annalen der Physik. 79: 361-376, 489-527; 80: 457-490; 81: 109-139. 
SchröDInGER, Erwin (1926b). "Über das Verhälnis der Heisenberg-BornJordanschen Quantenmechanik zu der meinen". Annalen der Physik. (4) 79: 734-756.

SchWARZSCHILD, Karl (1916). "Über das Gravitationsfeld eines Massenpunktes nach der Einsteinsche Theorie". K. Preussische Akademie der Wissenschaften. Sitzungsberichte. 1916: 189-196.

SitTer, Willem de (1917). “On Einstein's theory of gravitation, and its astronomical consequences. Third Paper". Monthly Notices of the Royal Astronomical Society. 76: 78: 3-28.

SLIPHER, Vesto M. (1913). "The radial velocity of the Andromeda Nebula". Lowell Observatory Bulletin. $\mathrm{N}^{\mathrm{o}} 58$.

SLIPHER, Vesto M. (1915). "Spectrographic observations of nebulae". Popular Astronomy. 23: 21-24.

Slipher, Vesto M. (1917). "Nebulæ". American Philosophical Society Proceedings. 56: 403-409.

StrömberG, Gustaf (1925). "Analysis of radial velocities of globular clusters and non-galactic nebulae". Astrophysical Journal. 61: 353-362.

Thomson, James (1884). "On the law of inertia, the principle of chronometry and the principle of absolute clinural rest, and of absolute rotation". Royal Society of Edinburgh Proceedings. 12: 568-578.

Tolman, Richard C. (1934). Relativity, Thermodynamics and Cosmology. Oxford: Clarendon Press.

Torretti, Roberto (1983). Relativity and Geometry. Oxford: Pergamon Press. (Second, corrected edition: New York, Dover, 1996).

TorretTi, Roberto (1994). La geometría del universo y otros ensayos de filosofía natural. Mérida: Comisión de Publicaciones de la Universidad de los Andes.

Torretti, Roberto (2003). Relatividad y espaciotiempo. Santiago de Chile: RIL editores. 2003.

Torretti, Roberto (2007). “'Rod contraction' and 'clock retardation': Two harmless misnomers?". En F. Minazzi, Filosofia, scienza e bioetica nel dibattito contemporaneo: Studi internazionali in onore di Evandro Agazzi. Roma: Istituto Poligrafico e Zecca dello Stato. pp. 1029-1039.

Torretti, Roberto (2008). Crítica filosófica y progreso científico: Cuatro ejemplos. Santiago de Chile: Ediciones Universidad Diego Portales 
ToRretTi, Roberto (2011). "Fenomenotecnia y conceptualización en la epistemología de Gastón Bachelard”. Theoria, 72: 97-114.

Torretti, Roberto (2013). Estudios filosóficos: 2010-2011. Santiago de Chile: Ediciones Universidad Diego Portales.

TucíDides (1942). Thucydidis historiae, 2 vols. Ed. H.S. Jones \& J.E. Powell. Oxford Clarendon Press, 1942.

von Neumann, John (1927). "Mathematische Begründung der Quantenmechanik". Nachrichten der Gesellschaft der Wissenschaften zu Göttingen. 1927: 1-57.

WIEN, Willy (1893). "Eine neue Beziehung der Strahlung schwarzer Körper zum zweiten Hauptsatz der Wärmetheorie". Preussische Akademie der Wissenschaften. Sitzungsberichte. pp. 55-62.

WiLson, Robert M. (1978). "The Cosmic Microwave Background Radiation". Nobel Lecture, 8 December, 1978. http://www.nobelprize.org/ nobel_prizes/ physics/laureates/1978/wilson-lecture.pdf. (Consultado el 26-03-2016). 
